\title{
Design of a Partially-Coupled Self-Adaptive Robotic Finger Optimized for Collaborative Robots
}

\author{
Lionel Birglen \\ Polytechnique Montreal \\ Montreal, QC, H3C 3A7, Canada \\ Email: lionel.birglen@polymtl.ca
}

\begin{abstract}
This paper presents the design and optimization of a self-adaptive, a.k.a. underactuated, finger targeted to be used with collaborative robots. Typical robots, whether collaborative or not, mostly rely on standard translational grippers for pickand-place operations. These grippers are constituted from an actuated motion platform on which a set of jaws is rigidly attached. These jaws are often designed to secure a precise and limited range of objects through the application of pinching forces. In this paper, the design of a self-adaptive robotic finger is presented which can be attached to these typical translational gripper to replace the common monolithic jaws and provide the gripper with shape-adaptation capabilities without any control or sensors. A new design is introduced here and specially optimized for collaborative robots. The kinetostatic analysis of this new design is briefly discussed and then followed by the optimization of relevant geometric parameters. Finally, a practical prototype attached to a very common collaborative robot is demonstrated. While the resulting finger design could be attached to any translational gripper, specifically targeting collaborative robots as an application allows for more liberty in the choice of design parameters as will be shown and the optimized parameters that are found take advantage of this property.
\end{abstract}

\section{INTRODUCTION}

Self-adaptive or underactuated hands and fingers as described in [4] have been successfully introduced throughout the robotics community, both in academia and the industry, as a middle ground solution between classical industrial grippers and complex anthropomorphic robotic hands. While keeping the control simplicity of the former, underactuated grasping allows for the shape adaptation of the latter to the complex surfaces of common objects. The application of underactuation to robotic hands relies on two complementary principles: first, a transmission mechanism is used to distribute a single actuation force or torque to the many joints of the (driven) finger; second, a set of passive elements are used to statically constrain the resulting mechanical device. The transmission mechanism can take the form of a linkage and, typically, preloaded springs maintaining the phalanges aligned when no contact has yet occurred serve as the passive elements. Notice however that the transmission mechanism can also be implemented using cables and pulleys as shown in maybe the oldest prototype of underactuated gripper, the Soft Gripper by Hirose and Umetani [10]. The number of phalanges of this latter device, namely ten, is rather peculiar and anthropomorphically inspired designs with three phalanges are much more common, e.g. the hands from Palli et al. [17], Dollar and Howe [8], Catalano et al. [7], Ozawa et al. [16]. When compared with fully actuated designs, underactuated fingers have an attractive simplicity since they typically require only one actuator, and do not rely on sensors or complex control scheme.

During the last decade, underactuated hands and fingers have migrated from university research centers to the industry and several companies have emerged to commercialize these end-effectors, such as Barrett Technology, Robotiq, Lacquey, and RightHand Robotics for the most well-known. Yet, the manufacturing and packaging industry still mostly relies on parallel grippers for their operations and is reluctant to replace them since they have been using these for decades sometimes. Indeed, the end-of-arm-tooling of choice for mechanical robotic manipulation seems to remain the classic parallel grippers (barring suction cups.) However, there is a solution to take advantage of the benefits of underactuated grasping while preserving the familiarity and know-how of the industry. This solution consists in using self-adaptive robotic fingers that can be attached to the usual industrial grippers in replacement of the monolithic jaws and thereby transforming the tool into a fully functional underactuated hand at a very low cost. This principle was embodied for the first time maybe in the FinGripper by Festo $\mathrm{GmbH}$ which was constituted by a compliant structure driven by the motion at its base. A more recent device based on a similar idea was presented by Carpenter et al. [6] where translational rods connected by pneumatic struts produced an effect similar to a spring-loaded bed of pins. Recently, Backus and Dollar [1] proposed a variation of a previously developed finger design that is similarly intended to be attached to a three-jaw concentric industrial gripper, although in this case another supplementary actuator is still required to initiate the enveloping motion of the finger.

Similarly, the author [2] presented a fully passive threephalanx self-adaptive mechanical finger capable of producing stable power (enveloping) and precision (pinch) grasps which ressembles the FinGripper but using rigid links. A prototype of this design, attached to an off-the-shelf translational pneumatic gripper, was successfully demonstrated in that reference. In the present paper, a refined and simplified version of this latter design is introduced and shown to be a valid alternative both theoretically and after experimentations. This novel design is also specifically dedicated to be used 
with collaborative robots (cobots) or more precisely, to be attached to their translational grippers and takes advantage of the intrinsic safety of these cobots to alleviate design requirements. These cobots, are designed for human-robot collaboration, i.e. working in close proximity to humans. Amongst the most well-known and successful cobots, one can find: the Universal Robots UR series, Fanuc's CR-35iA, Kuka LWR series, Rethink's Baxter and Sawyer, ABB's Yumi, and Gomtec's Roberta series (note: Gomtec was recently purchased by ABB.) It should be noted that the latter three manufacturers all provide standard translational grippers with their collaborative robots. Finally, in a recent paper Franchi et al. [9] also showed an adaptive finger relying on the translational gripper of the Baxter robot to provide actuation but that design required physical modifications of the gripper conversely to the solution proposed here.

\section{Kinetostatic Analysis}

The design of self-adaptive finger previously presented in [2] was based on a sixbar linkage with revolute joints. Four consecutive links of this mechanism were chosen as the ground and phalanges of the finger. The remaining two links constituted the transmission linkage distributing the passive element torques (i.e. from the springs) to the phalanges. The three revolute joints of these two links were indeed required in the transmission linkage to avoid constraining the DOF of the finger and ensure maximal shape-adaptivity to the object seized. However, as will be shown in this paper having a transmission linkage providing full mobility to the phalanges is arguably not critical to ensure a successful grasp as evidenced by experimental results, see Section IV A simplified design with only two revolute joints in the transmission linkage is proposed here. This simplified design is illustrated in Fig. 1. The translational gripper on which the finger is attached is modeled by the prismatic joint at the base of the mechanism. When this joint is driven, a contact between the proximal or intermediate phalanges and the object that is to be seized creates a movement in the phalanx joints $O_{1}^{F}, \ldots, O_{3}^{F}$. These phalanges are initially constrained in the fully upright position by the springs in $O_{1}^{T}$ and $O_{2}^{T}$, respectively providing a torque $\mathbf{t}_{1}=t_{1} \mathbf{z}$ and $\mathbf{t}_{2}=t_{2} \mathbf{z}$.

It should be noted that while the finger has three phalanges, the whole mechanism (finger+transmission linkage) constitutes a fivebar linkage and thus, has only two DOF. This implies that:

1) there is a coupling between the phalanx motions,

2) only two contacts are required to statically constrain the finger.

Both of these properties yield important consequences on the kinetostatic analysis of the finger and its performance in terms of forces and workspace, as will be shown.

Force Analysis: In order to establish the forces that this new design can apply onto objects, a kinetostatic analysis is proposed here following the same methodology and notations

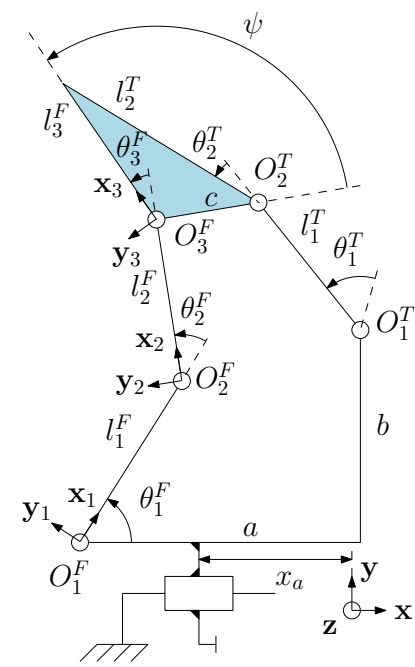

(a) Geometry

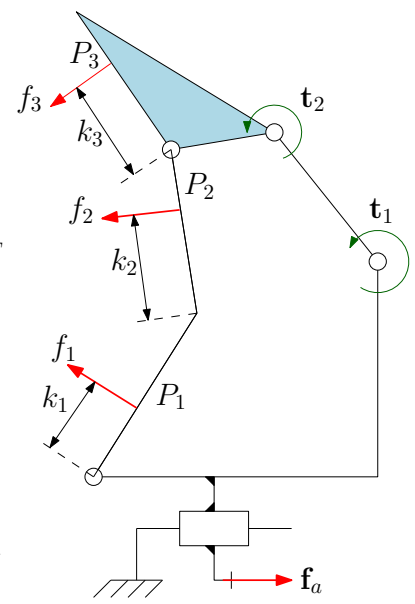

(b) Force/Torque
Fig. 1. Parameters of the novel simplified mechanism: joints $O_{1}^{F}$ to $O_{3}^{F}$ define the phalanges, joints $O_{1}^{T} / O_{2}^{T}$ the transmission linkage, $f_{a}$ and the translation stand for the robot gripper. Contacts can occur at points $P_{1}$ to $P_{3}$ and springs create torques $\mathbf{t}_{1}$ and $\mathbf{t}_{2}$

as these used in [2] which will also allow for a comparison with the latter reference. It is well-known that adaptive fingers cannot always generate positive contact forces at all phalanges. If one contact force becomes negative, the finger will reconfigure itself on the object's surface until it either reaches a stable configuration or loses contact with the object. While the former phenomenon is far more common than the latter, degeneration of the contact configuration due to negative contact forces has been shown to be very possible. To establish these contact forces, assuming that dynamic forces are negligible, the virtual work principle can be used and yields:

$$
\delta W=\mathbf{f}_{a}^{T} \boldsymbol{\delta} \mathbf{x}_{a}+\mathbf{f}_{i}^{T} \boldsymbol{\delta} \mathbf{y}^{i}+\mathbf{t}^{T} \boldsymbol{\delta} \boldsymbol{\theta}_{T}=0
$$

where $\mathbf{f}_{a}=f_{a} \mathbf{x}$ is the force associated with the actuation of the finger itself corresponding to a translation along the $\mathrm{x}$-axis. The infinitesimal motion of the actuator is then $\delta \mathbf{x}_{a}=\delta x_{a} \mathbf{x}$. In this paper, the notation $\delta x$ and $\delta \mathbf{x}$ stands for an infinitesimal variation of, respectively, the scalar $x$ and the vector $\mathbf{x}$. The torques due to the springs are modeled with the vector $\mathbf{t}=$ $\left[\begin{array}{ll}t_{1} & t_{2}\end{array}\right]^{T}$ and the rotations associated with these torques, i.e. the relative joint angles in the transmission linkage, are grouped in vector $\boldsymbol{\theta}_{T}=\left[\begin{array}{ll}\theta_{1}^{T} & \theta_{2}^{T}\end{array}\right]^{T}$. The vector $\mathbf{f}_{i}$ represents the contact forces generated by the finger at the phalanges and depends on the contact scenario $i$. Indeed, since only two contact points are sufficient to fully constrain the finger, three cases or contact scenarios, illustrated in Fig. 2, must be studied. Case \# $i$ is defined as a situation where the contact force $f_{i}$ is missing. The contact forces themselves are assumed to be normal to the surface of the phalanges, i.e. friction is neglected, and acting along a vector $\mathbf{y}_{k}$ with $k=1,2,3$, and the vector $\boldsymbol{\delta} \mathbf{y}^{i}$ is 
defined by:

$$
\boldsymbol{\delta} \mathbf{y}^{i}=\left[\begin{array}{c}
\delta \mathbf{r}_{P_{j}}^{T} \mathbf{y}_{j} \\
\delta \mathbf{r}_{P_{k}}^{T} \mathbf{y}_{k}
\end{array}\right] \quad \text { with }(j, k) \neq i
$$

where $\mathbf{r}_{P_{m}}$ is the vector from point $O$ to the contact point $P_{m}$

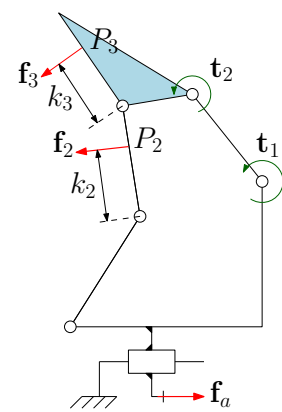

(a) case \#1

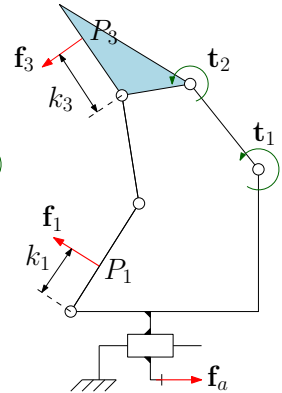

(b) case \#2

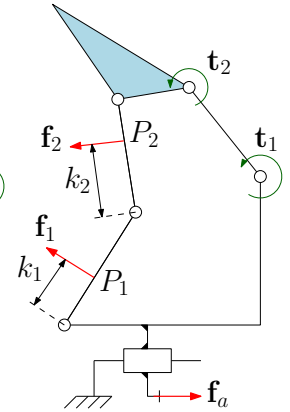

(c) case \#3
Fig. 2. Contact scenarios

which is itself at a distance $k_{m}$ from the base of the associated phalanx. Neglecting friction might at first be seen as a highly impractical hypothesis for a robotic finger and frictional pads are indeed covering most existing prototypes, including the one presented in Section IV] However, as shown in [3, 14], friction improves the grasp stability of underactuated fingers. Thus, neglecting it is actually a conservative hypothesis and the real performance of a finger designed thusly is expected to exceed that of the model. Taking into account friction at the design stage also requires to select the properties at the contact points (a pair of materials for instance) which are dependent on the application.

By choosing the three DOF of the mechanism (two for the finger, one for the actuation) as the translation $x_{a}$ and the angles $\theta_{1}^{F}$ and $\theta_{2}^{F}$, a Jacobian matrix $\mathbf{J}_{i}$ can be defined for each contact scenario as:

$$
\left[\begin{array}{c}
\delta x_{a} \\
\delta \mathbf{y}_{F}^{i}
\end{array}\right]=\mathbf{J}_{i}\left[\begin{array}{c}
\delta x_{a} \\
\delta \boldsymbol{\theta}_{F}
\end{array}\right]
$$

where $\boldsymbol{\theta}_{F}=\left[\begin{array}{ll}\theta_{1}^{F} & \theta_{2}^{F}\end{array}\right]^{T}$. When the transmission linkage of the finger had three revolute joints, the Jacobian matrix is the same as the one defined in [2], namely:

$$
\mathbf{J}=\left[\begin{array}{cccc}
1 & 0 & 0 & 0 \\
-s_{1} & k_{1} & 0 & 0 \\
-s_{12} & l_{1} c_{2}+k_{2} & k_{2} & 0 \\
-s_{123} & l_{1} c_{23}+l_{2} c_{3}+k_{3} & l_{2} c_{3}+k_{3} & k_{3}
\end{array}\right]
$$

where $s_{i \ldots j}$ and $c_{i \ldots j}$ are shorthand notations for respectively $\sin \left(\sum_{k=i}^{j} \theta_{k}^{F}\right)$ and $\cos \left(\sum_{k=i}^{j} \theta_{k}^{F}\right)$.

Partial Coupling: Based upon these definitions and conversely to the previous reference, the matrices $\mathbf{J}_{i}$ can be defined for each contact scenario simply by removing the $i+1$ line of $\mathbf{J}$, i.e. the line corresponding to the missing $\delta \mathbf{y}^{i}$ of the scenario. Then, one can relate $\boldsymbol{\delta} \boldsymbol{\theta}_{F}$ and $\boldsymbol{\delta} \boldsymbol{\theta}_{F}^{i}$ through a coupling matrix $\mathbf{C}_{i}$ defined for each contact scenario $i$ by:

$$
\left[\begin{array}{c}
\delta x_{a} \\
\boldsymbol{\delta} \boldsymbol{\theta}_{F}
\end{array}\right]=\mathbf{C}_{i}\left[\begin{array}{c}
\delta x_{a} \\
\boldsymbol{\delta} \boldsymbol{\theta}_{F}^{i}
\end{array}\right] .
$$

These matrices can be expressed as:

$$
\begin{gathered}
\mathbf{C}_{1}=\left[\begin{array}{ccc}
1 & 0 & 0 \\
0 & X_{1} & Y_{1} \\
0 & 1 & 0 \\
0 & 0 & 1
\end{array}\right] \quad \mathbf{C}_{2}=\left[\begin{array}{ccc}
1 & 0 & 0 \\
0 & 1 & 0 \\
0 & X_{2} & Y_{2} \\
0 & 0 & 1
\end{array}\right] \\
\mathbf{C}_{3}=\left[\begin{array}{ccc}
1 & 0 & 0 \\
0 & 1 & 0 \\
0 & 0 & 1 \\
0 & X_{3} & Y_{3}
\end{array}\right]
\end{gathered}
$$

where the coefficients $X_{i}$ and $Y_{i}$ are relating the motion of the phalanx joint angle where a contact is missing to the other two phalanx joint angles. These matrices can be obtained by considering the kinematics of the two fourbar linkages defined by $O_{i}^{F} O_{k}^{F} O_{1}^{T} O_{2}^{T}$ with $k \neq i$. For example, if $i=1$ one obtains from Eqs. (5)-(6):

$$
\delta \theta_{1}^{F}=X_{1} \delta \theta_{2}^{F}+Y_{1} \delta \theta_{3}^{F} .
$$

A differential form can be immediately recognized with:

$$
\left\{\begin{array}{l}
X_{1}=\frac{\delta \theta_{1}^{F}}{\delta \theta_{2}^{F}} \text { for } \delta \theta_{3}^{F}=0 \\
\text { and } Y_{1}=\frac{\delta \theta_{1}^{F}}{\delta \theta_{3}^{F}} \text { for } \delta \theta_{2}^{F}=0
\end{array}\right.
$$

and thus, $X_{1}$ can be computed by considering the $O_{1}^{F} O_{2}^{F} O_{1}^{T} O_{2}^{T}$ fourbar while $Y_{1}$ requires to use $O_{1}^{F} O_{3}^{F} O_{1}^{T} O_{2}^{T}$. The reader is then referred to the literature for analytic methods to obtain the velocity relationship between the angles of a fourbar linkage, e.g. in the textbook by McCarthy [15]. Combining Eqs. (3) and (6), one then obtains:

$$
\left[\begin{array}{c}
\delta x_{a} \\
\delta \mathbf{y}_{F}^{i}
\end{array}\right]=\mathbf{J}_{i} \mathbf{C}_{i}\left[\begin{array}{c}
\delta x_{a} \\
\delta \boldsymbol{\theta}_{F}^{i}
\end{array}\right] .
$$

A last matrix is required to completely characterize the grasps of the finger, namely a Transmission matrix $\mathbf{T}_{i}$, relating the joint angles of the transmission linkage (hence its name) to the vector in the righthand side of Eq. 99, i.e.:

$$
\delta \boldsymbol{\theta}_{T}=\mathbf{T}_{i}\left[\begin{array}{c}
\delta x_{a} \\
\delta \boldsymbol{\theta}_{F}^{i}
\end{array}\right] .
$$

This matrix can be easily established from the Transmission matrix of the original design of the mechanism presented in [2] by removing the last line of the matrix as well as the $i^{e}$ column. Finally, combining Eqs. (9) and (10) to Eq. (1) yields:

$$
\mathbf{f}=-\left(\mathbf{J}_{i} \mathbf{C}_{i}\right)^{-T} \mathbf{T}_{i}^{T} \mathbf{t} \quad \text { with } \mathbf{f}=\left[\begin{array}{c}
f_{a} \\
\mathbf{f}_{i}
\end{array}\right]
$$

where $\mathbf{X}^{-T}$ is the transpose and inverse of matrix $\mathbf{X}$. Once the contact scenario is defined based on the shape and position of an object to seize, this equation allows to compute the magnitudes of the contact forces at the phalanges as well as the required force of the translational gripper necessary 
to maintain the mechanism in static equilibrium. This is obviously very useful to optimize the grasping performance of the finger before building a prototype.

\section{DESIGN OPTIMIZATION}

With the results of the previous section one can optimize a design of the proposed mechanism. The first question to answer is to decide what needs to be optimized. There have been quite a few performance criteria proposed in the literature, most of which have been enumerated by Kragten and Herder [13], depending on which technology is used to drive the finger (cables, linkages, deformable structure, etc.) Here, the percentage of a target workspace where all the generated contact forces are positive is chosen to be used. Indeed, as mentioned earlier, some contact force magnitudes as computed from Eq. (11) might be negative. If such a situation arises in practice the phalanx for which this occurs will move away from the object, causing a sliding motion of the finger along the object generally until a mechanical limit is reached. Alternatively, with really poor designs, if no mechanical limit prevents this reconfiguration of the finger, the sliding will continue until the object is ejected from the finger. Using naive design parameters, an example of the typical force workspace of the mechanism proposed in this paper is illustrated in Fig. 3 for contacts at mid-phalanx and unitary stiffnesses of the springs. In this example, different undesired areas are clearly visible where:

1) the design cannot be assembled (links are too short),

2) at least one contact force is negative,

3) mechanical limits would be reached.

It can be clearly seen that maximizing the area where all contact forces are positive would be desirable and also lead to increasing the reachable workspace of the finger.

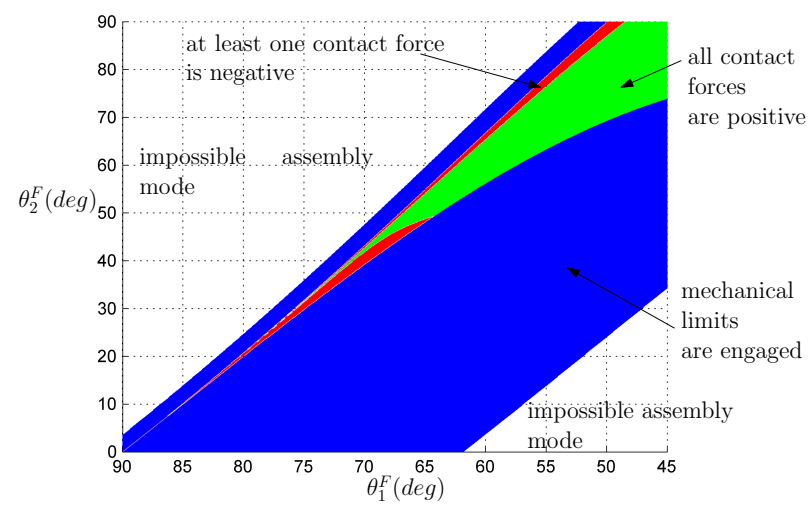

Fig. 3. Example of a typical force workspace for a finger design

Optimization Function: Mathematically, the capability of the proposed mechanism to create positive contact forces can be measured by:

$$
\mu=\frac{1}{3} \sum_{i=1}^{3} \frac{\int_{W} \delta^{i}(\boldsymbol{\theta}) d \boldsymbol{\theta}}{\int_{W} d \boldsymbol{\theta}}
$$

where $W$ is the workspace of the finger in terms of phalanx joint angle ranges and $\delta^{i}(\boldsymbol{\theta})$ is a Kronecker symbol for the positiveness of the contact forces in the contact scenario $i$ equals to one if all $f_{k} \geq 0(k \neq i)$ or 0 otherwise. Note that $\mu$ is dimensionless and has a maximal value of 1 .

The capability of an underactuated finger to generate positive contact forces over its workspace is but one optimization metric that can be used. Several other indices have been discussed, see the work by Kragten and Herder [13] again. For instance, typical objects (generally cylinders) can be considered instead of the whole workspace, as in [5] for example, or the resulting stability of the grasped objects can be studied as shown by Kragten et al. [12]. However, the actual ability to generate contact forces might be most basic requirement for a robotic finger and since no particular objects or applications were a priori considered, Eq. (12) appeared an acceptable choice to measure performance.

Now, the design parameters available for the optimization must be chosen. To simplify the problem, the phalanx lengths were chosen to be $l_{1}^{F}=l_{2}^{F}=30 \mathrm{~mm}$ and $l_{3}^{F}=45 \mathrm{~mm}$. These values correspond to a 0.75 scaled down version of the prototype presented in [2] which used a significantly larger translational gripper. The rationale for this rescaling is that the prototype developed in this paper is intended to be attached to a Baxter robot and it was desirable that the overall length of our finger would not be larger than the longest of the standard jaws provided with the electric gripper of this robot, namely $109 \mathrm{~mm}$. However, by keeping the ratio of phalanx lengths identical, the comparison is fair.

Therefore, only four parameters remain:

1) $a$ : horizontal distance between $O_{1}^{F}$ and $O_{1}^{T}$,

2) $b$ : vertical distance between $O_{1}^{F}$ and $O_{1}^{T}$,

3) $c$ : distance between $O_{3}^{F}$ and $O_{2}^{T}$,

4) $\psi$ : solid angle defining the distal phalanx.

See Fig. 1 where all these design variables are illustrated. Of these, the angle $\psi$ has to be set to $\pi / 2$ in order for stable pinch grasps to be achievable. Indeed, by adding mechanical limits in the joints at points $O_{1}^{T}$ and $O_{2}^{T}$ preventing the latter to rotate clockwise, passive stable pinch grasps can be achieved without interfering with the capability of offering enveloping grasps as illustrated in Fig. 4 For this, one must make sure that any contact on the distal phalanx will create a clockwise rotation in both joints. The simplest way to do that is by making sure that a contact force there is above $O_{2}^{T}$, and thus $\psi \geq \pi / 2$. However, if $\psi$ is greater than $\pi / 2$, any contact on the intermediate phalanx whose line of action is above $O_{2}^{T}$ would also be opposed by the mechanical limits thereby preventing the enveloping motion of the first two phalanges. To avoid this, one is left with no choice but $\psi=\pi / 2$.

Design for Collaborative Robots: In the end, three geometric parameters are available for the optimization: $a, b$, and $c$. A summary of the fixed and variable parameters in the design is presented in Table I. The chosen ranges of the design parameters are also indicated in this Table. These ranges were 


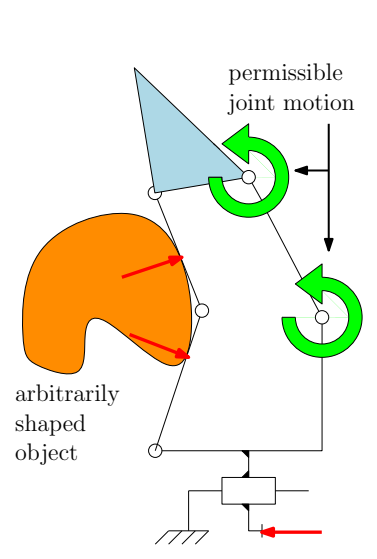

(a) power

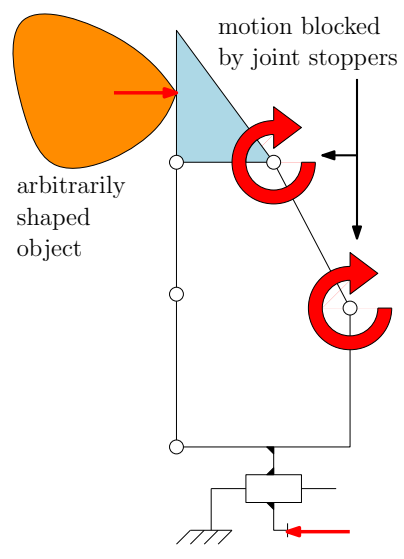

(b) precision
Fig. 4. Power and precision grasps achievable using mechanical limits

TABLE I

GEOMETRIC PARAMETERS FOR THE OPTIMIZATION OF THE FINGER (ALL LENGTHS ARE IN MM).

\begin{tabular}{||l||c|c|c|c|c|c|l|}
\hline Parameter & $l_{1}^{F}$ & $l_{2}^{F}$ & $l_{3}^{F}$ & $a$ & $b$ & $c$ & $\psi$ \\
\hline Values/ranges & 30 & 30 & 45 & {$[10,60]$} & {$[0,50]$} & {$[10,60]$} & $\pi / 2$ \\
\hline
\end{tabular}

chosen to avoid mechanical interferences between the links of the mechanism and with the translational gripper. It should also be noted that one attractive feature of collaborative robots is the ability to safely accommodate collisions. Therefore, while it was necessary in [2] to have the lowest joint of the transmission linkage as close as possible to the base (i.e. $b=0$ ) to make the finger collision-safe, this requirement can be relaxed here since the robot is detecting collision by itself and stopping before potentially damaging forces arise. This is one of the main reason why the design proposed here is said to be targeted for cobots: collisions with the finger are not a concern and do not yield additional design constraints. While taking advantage of the inherent safety of cobots, it should be pointed out that the finger optimized here is not intended specifically for collaborative tasks (i.e. in close proximity to a human operator) per se although a particular attention has been paid out when designing the actual prototype described in Section IV to the avoidance of sharp edges and pinch points in accordance with the recent norm ISO/TS 15066:2016 [11]. To mitigate the danger of pinch points, the width of the inside hole of the finger (the area of the inscribed polygon $O_{1}^{F} O_{2}^{F} O_{3}^{F} O_{2}^{T} O_{1}^{T}$ ) should be kept small to prevent a human to put his/her own finger inside the mechanism. This requirement contrasts with the initial design presented in [2] where this property was neglected.

Results: An example of the values of the performance index $\mu$ as functions of $a$ and $b$ for different $c$ is illustrated in Fig 5 . The contact forces were evaluated for contacts at mid-phalanges and assuming springs of equal and unitary stiffnesses in joints
$O_{1}^{T}$ and $O_{2}^{T}$. The workspace of the finger was defined by:

$$
W \equiv\left\{\begin{array}{l}
\pi / 4 \leq \theta_{1}^{F} \leq \pi / 2 \\
0 \leq \theta_{2}^{F} \leq \pi / 2
\end{array}\right.
$$

The best performance possible for a design as a function of $c$ is illustrated in Fig. 6 Note that in the latter Fig., a minimal value of $c=5 \mathrm{~mm}$ was used to clearly show the peak around $c=12 \mathrm{~mm}$. From these results, one can clearly see that it is beneficial to keep $c$ small. However, values below $15 \mathrm{~mm}$ are difficult to design as the axes and housings of the joints at $O_{2}^{T}$ and $O_{3}^{F}$ tend to interfere. In practice, and again to avoid mechanical interference, a value of $15 \mathrm{~mm}$ for $c$ was selected. As can clearly be seen in Fig. 5, large values of $a$ are typically preferable for most values of $c$. However, in our case with $c=15 \mathrm{~mm}$, this would lead to a very bulky design. There is therefore a trade-off to be made between performance and compactness. Taking as a measure of the latter the value of $a$, i.e. the width of the finger, the optimal value of $b$ for each $a$ as well as the Pareto front (trade-off curve) between $a$ and $\mu$ is illustrated in Fig. 7. Dynamic simulations were also conducted using MSC ADAMS ${ }^{\mathrm{TM}}$ as illustrated in Fig. 8 Notice how in this figure how the design with a smaller $a$ both leads to mechanical interference as well as a significantly smaller contact force at one phalanx. This small force is on the verge of becoming negative (vanishing) conversely to the second design (larger $a$ ) which shows much more balanced forces. As a final choice, it was decided to keep $a$ at $25 \mathrm{~mm}$ to have to compact design. Then, looking at Fig. 7 , the best value for $b$ was $31 \mathrm{~mm}$ which gives a final performance index of $\mu=0.07$. As a comparison, the absolute best design with the parameter ranges listed in Table 1 reached a performance index of 0.11. A degradation in grasp performance was therefore accepted for the sake of an improvement in the compactness performance. However, selecting these geometric parameters results has another benefit: the actual distance between the phalanges of the finger and the transmission linkage is then $18 \mathrm{~mm}$ considering the practical widths of the links (see the prototype in Section IV]. This distance is smaller than the value of one digit (the unit), i.e. the average breadth of the human finger $(19 \mathrm{~mm})$, and thus, the risk of a human finger to get stuck in the linkage while in operation, if not completely eliminated as it is only an average value, is relatively limited. It should also be noted that the forces developed by the Baxter electric gripper are small enough for this issue to not be a major concern, see Section IV again for actual numbers, and therefore, larger widths were considered acceptable during the optimization.

Another comparison could be made with the (not simplified) design with non-coupled phalanges presented in [2] which reached a value of 0.10 with a similarly defined performance index. Although in that case, the comparison is not entirely accurate since we are comparing between indexes computed from three two-dimensional workspaces (here) to one that is three-dimensional (non-coupled phalanges), it seems to indicate that the diminished grasp performance obtained by 


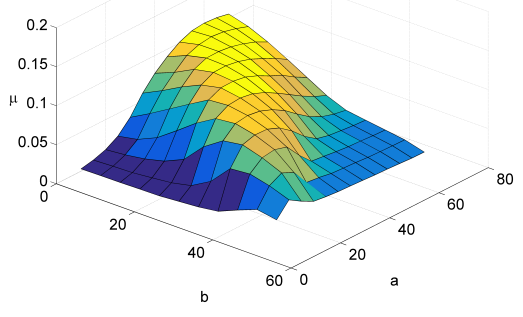

(a) $c=10$

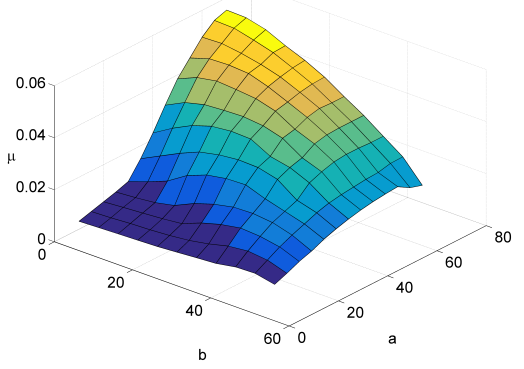

(d) $c=40$

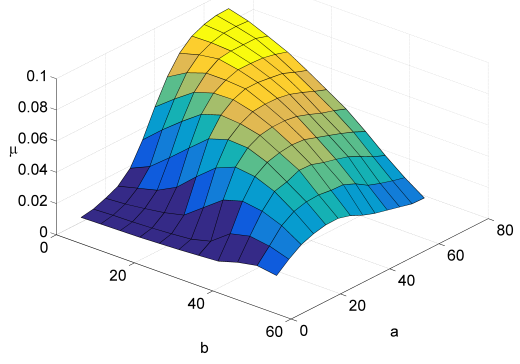

(b) $c=20$

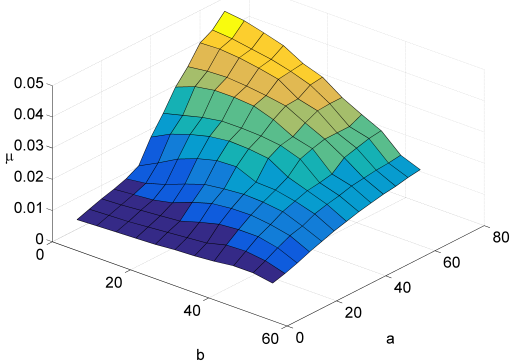

(e) $c=50$

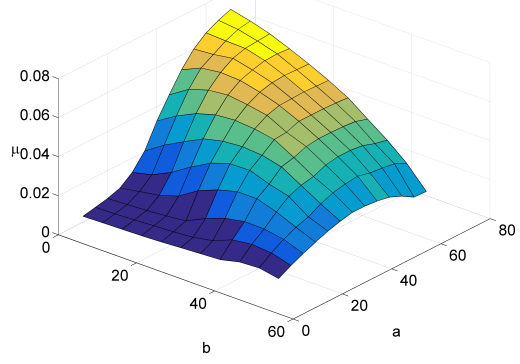

(c) $c=30$

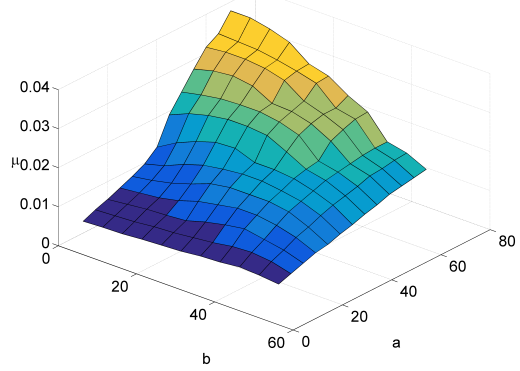

(f) $c=60$

Fig. 5. Performance index as a function of $a$ and $b$ for different values of $c$ (all values of $a$ and $b$ are in mm)

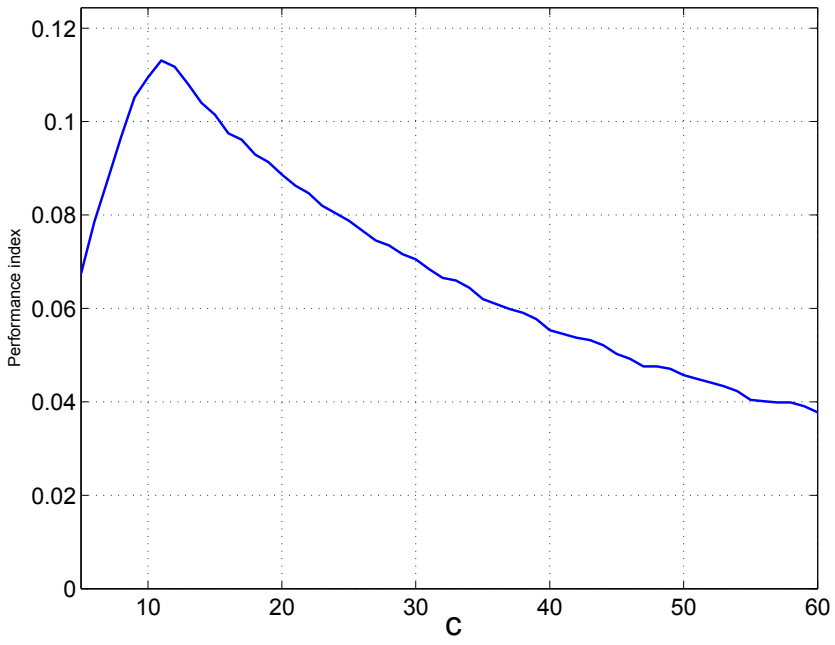

Fig. 6. Best performance index possible as a function of $c(\mathrm{~mm})$

using the partially coupled design presented here remains limited and if compactness is not a concern, an even slightly better performance can be achieved. That is, the percentage of the finger workspace with fully positive contact forces can be approx. 10\% larger with the coupled design (the performance index goes from 0.10 to 0.11 ). This strengthens the argument proposed by this paper that a full-mobility adaptive finger might not be required. However, if theoretical properties appear encouraging, actual physical experiments are required to ensure that the introduction of a coupling and therefore, reduced mobility for the finger is not detrimental to the capability of grasping various objects. This last point will be clearly illustrated in the next and final Section.

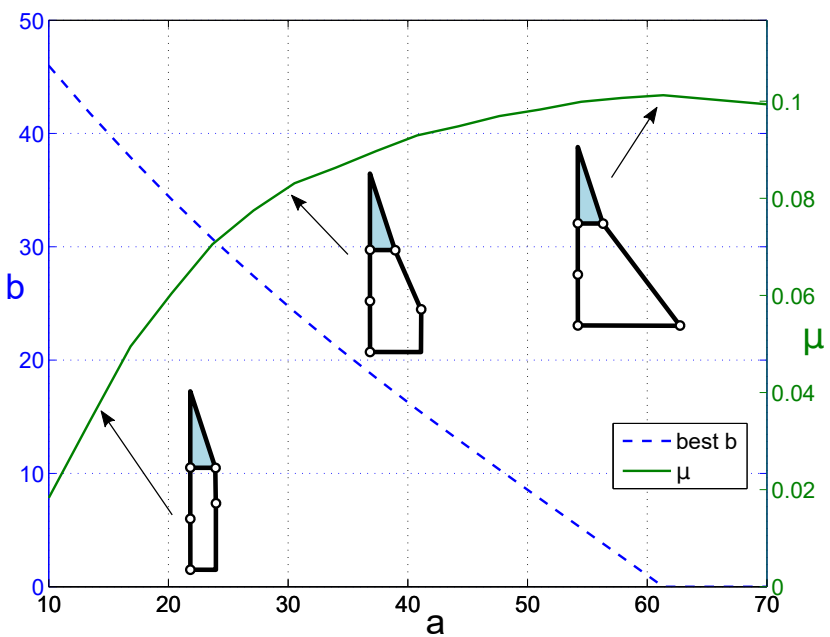

Fig. 7. Optimal values of $b$ for each $a$ (dashed blue) and Pareto front $(\mu)$ of the optimization, both for $c=15 \mathrm{~mm}$. Large values of $a$ lead to bulkier designs, the trade-off between compactness and performance is illustrated by the Pareto curve (solid green) indicating the degradation of $\mu$ with the improvement of $a$ (lower values of $a$ equal better compactness but lead to lower values of $\mu$.) 


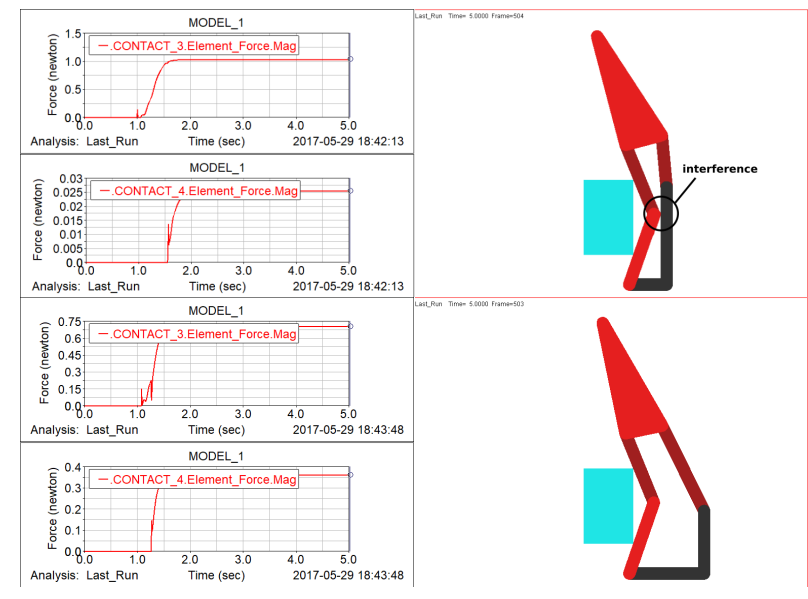

Fig. 8. Dynamic simulations of grasps with contact force measurements

\section{EXPERIMENTS}

Design: Finally, a prototype was built based on the optimal design previously found. The adaptive fingers were built with a fused deposition modeling rapid prototyping machine in a material with properties very similar to ABS. The springs were made from CS70 high carbon spring steel (zinc plated) with a zero-load length of $25.4 \mathrm{~mm}$ and a diameter of $5.6 \mathrm{~mm}$. Their measured linear stiffness was $4.3 \mathrm{~N} / \mathrm{mm}$. The inside surfaces of the fingers were covered with a thin $(0.8 \mathrm{~mm})$ neoprene cover with a Shore $40 \mathrm{~A}$ durometer to provide increased adherence which is helpful to oppose the pulling of seized object in the direction perpendicular to the plane of action of the fingers ( $\mathbf{z}$ axis in Fig. 1). These neoprene sheets have a typical dry static friction coefficient ranging from 1.4 to 2.2 with typical materials such as glass and steel according to the ASTM D-1894 specifications and are easy to cut which make them a popular choice for applications where increased friction is desirable such as grasping.

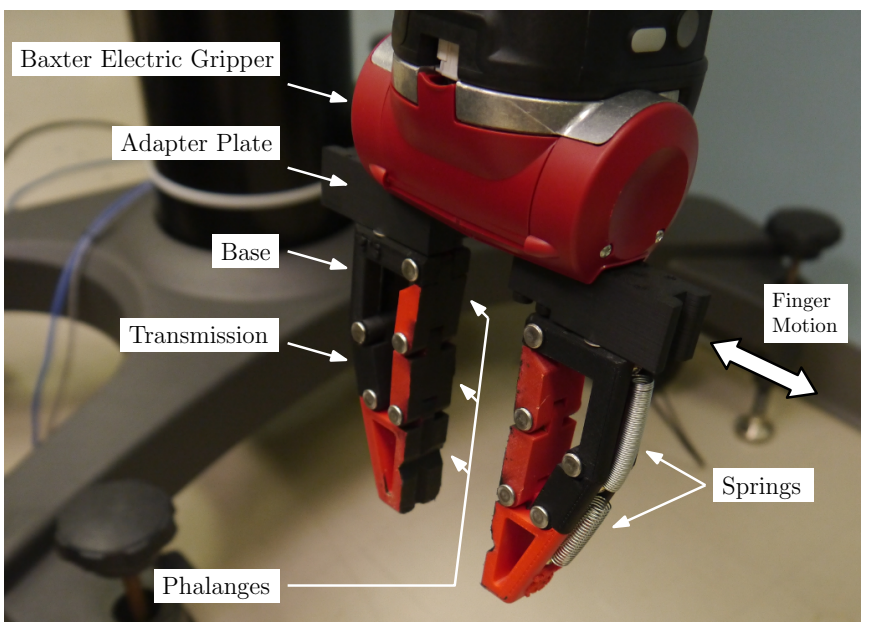

Fig. 9. Prototype of adaptive fingers mounted on the electric gripper of a Baxter robot

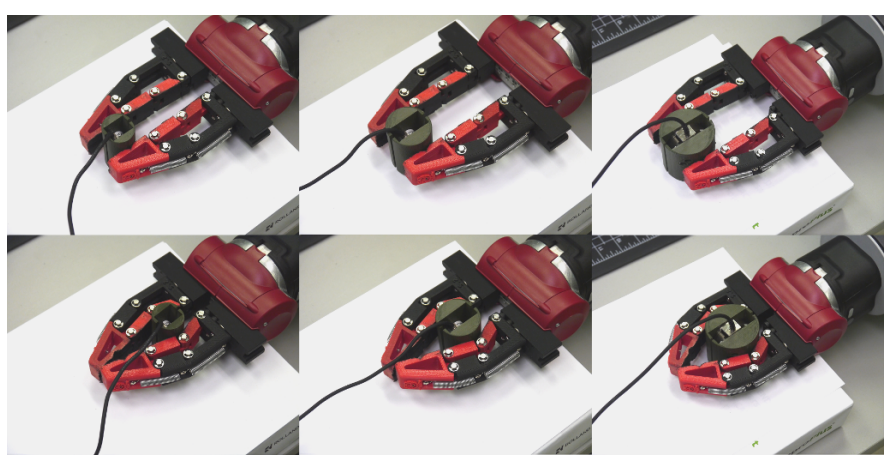

Fig. 10. Power and precision graps of cylindrical objects with force sensors

The actual prototype is illustrated in Fig. 9 and was found to work well for a variety of objects both in power and precision grasps. The range of motion of the fingers is dictated by the translation range of the Baxter electric gripper namely $20 \mathrm{~mm}$ for each finger which makes the whole hand (translational gripper + adaptive fingers) able to grasp objects in the range of 0 to $40 \mathrm{~mm}$ although very small objects (think of a needle) can be impractical to seize with an enveloping grasp due to clearance in the joints of the mechanism. In practice, the minimal size of the objects as projected along the translational direction to ensure a safe and secure grasp was found to be around $5 \mathrm{~mm}$. As a comparison, Robotiq two- and three-finger adaptive grippers require a minimal size of 20 to $43 \mathrm{~mm}$ for enveloping grasps according to their specifications. Minimal object sizes do not seem to be available in the literature for other commercial grippers such as the BarrettHand or FinGripper. It should be noted that smaller objects such as the aforementioned needle could still be efficiently seized with the fingers proposed here but using a pinch grasp similarly to the way humans seize small objects.

Measurements and Comparisons: The adaptive fingers proposed here can be attached in several different positions on the adapter plate (see Fig. 97 so while the total motion span is always $40 \mathrm{~mm}$, the grasping range can be 10 to $50 \mathrm{~mm}$, or 20 to $60 \mathrm{~mm}$, etc. up to 40 to $80 \mathrm{~mm}$. Greater ranges could be obtained with longer adapter plate even, if required. Three pairs of half-cylindrical shells were 3D printed and embedded with 6-axis force sensors, namely either an ATI-IA NANO17 or MINI45, as illustrated in Fig. 10 to measure the grasping forces. The results are listed in Table III along with the cylinder diameters. Each number in this Table corresponds to the magnitude of the squeezing force (i.e., the sum of all the individual contact forces) and is an average obtained from 10 tries (typical results are within $\pm 5 \%$.) Note that for the $52 \mathrm{~mm}$ cylinder, the position of the fingers was chosen for the hand to have a grasp range of 20 to $60 \mathrm{~mm}$ (illustrated in Fig. 10 rightmost column.)

One can see from Table III that the measured forces are quite similar and all between 16 to $18 \mathrm{~N}$, to be compared to a range of 4 to $7.5 \mathrm{~N}$ reported by Franchi et al. [9] which latter gripper has however a greater sweeping range that the one of 
TABLE II

MEASURED AVERAGE GRASPING FORCES.

\begin{tabular}{|r|c|c|c|}
\hline Cylinder diameter (mm) & 18 & 35 & 52 \\
\hline Sensor Model & \multicolumn{2}{|c|}{ Nano17 } & Mini45 \\
\hline Power Grasp Force (N) & 15.90 & 15.29 & 15.07 \\
\hline Pinch Grasp Force (N) & 18.17 & 17.25 & 16.37 \\
\hline
\end{tabular}

the fingers here. Precision graps are approximetely 10-15\% stronger that power grasps for our adaptive fingers since for enveloping grasps, part of the electric gripper force is used to flex the springs in the transmission linkage, reducing the available output contact forces. This emphasizes the need to keep this compliance as small as possible. Pinch forces measured with the original Baxter monolithic fingers are the same as the pinch forces measured with the fingers proposed here since during this type of grasp, the latter are essentially the same as the former, cf. Fig. 4(b) However, resistance to extraction of the object using Baxter's original fingers is much more limited since in that case only two opposing forces are available and pulling in the direction perpendicular to these two contact forces is only opposed by friction unless custom padding is added. Conversely, the fingers proposed here can envelope a wide range of objects. This envelopment leads to several contact points and thus, forces with different directions which can oppose a perturbation coming from a wider range of possible orientations.

A typical example of the force measured during a grasp and release of one sensorized cylinder is illustrated in Fig. 11. It can be seen that if the power grasp is slightly slower to get to a steady-state value due to the time required for the fingers to flex around the object, this remains marginal (from $70 \mathrm{~ms}$ to $98 \mathrm{~ms}$.) A video demonstrating the capabilities of the hand for a set of five random objects (three were picked from the 2016 Amazon Bin Picking Challenge list, two from general household items) and showing both types of grasps can be found at: http://youtu.be/neoeVbGLkF0 while still frames from this video are shown in Fig. 12. The video shows the Baxter robot seizing two cylinders of different diameters with a power grasp as well as one rectangular box and also pinching two objects, one of which is deformable. It illustrates how the fingers seize each object effectively. It can also be noted that in the case of the enveloping grasp of the rectangular box (video only), the proximal and intermediate phalanges rotate only slightly before reaching a stable configuration, conversely to when grasping cylinders. However, in both cases a static equilibrium is reached leading to a secure hold. Each one of these grasps was programmed offline since grasp planning is beyond the scope of this paper.

\section{CONCLUSIONS}

This paper presented a novel type of adaptive mechanical fingers that can be attached to the standard translational gripper of collaborative robots and transform this gripper into a fully functional underactuated hand. This design shows a simplified
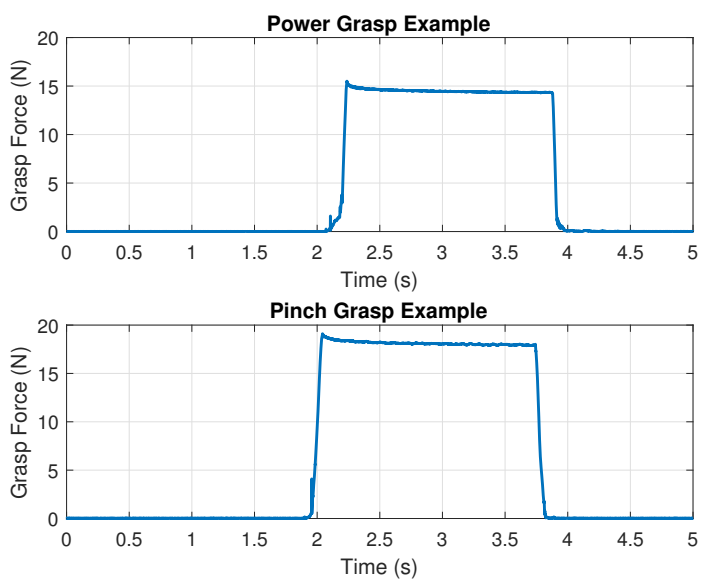

Fig. 11. Total grasp force measured by the force sensor during a grasp and release sequence (18 $\mathrm{mm}$ cylinder)

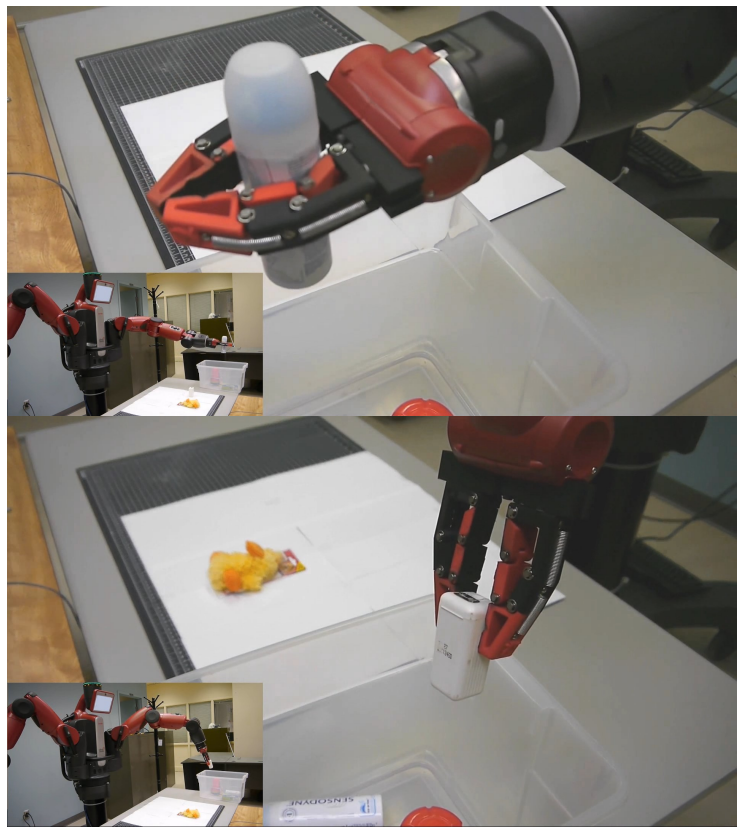

Fig. 12. Packing a box with random objects using power and precision grasps (still frames from the linked video)

design compared to the geometry of previous prototypes aiming at full mobility but, as illustrated here both from theoretical and practical results, its performances appear to be at least comparable. A prototype was built and experimented with. It was shown to be able to successfully grasp and securely hold during manipulation a wide range of objects. The important lesson learned here was that simplifying the linkage appears to be a reasonable trade-off as evidenced by the results obtained and, in particular, the practical experiments.

\section{ACKNOWLEDGMENTS}

The help of Dmitri Fedorov for the programming of the Baxter robot used for demonstration is gratefully acknowledged. The support from the Natural Sciences and Engineering Research Council (grant RGPIN327005) is also noted. 


\section{REFERENCES}

[1] S. B. Backus and A. M. Dollar. An Adaptive ThreeFingered Prismatic Gripper With Passive Rotational Joints IEEE Robotics and Automation Letters, 1(2):668675, July 2016. ISSN 2377-3766. doi: 10.1109/LRA. 2016.2516506.

[2] L. Birglen. Enhancing versatility and safety of industrial grippers with adaptive robotic fingers. In 2015 IEEE/RSJ International Conference on Intelligent Robots and Systems (IROS), pages 2911-2916, Sept 2015. doi: 10.1109/IROS.2015.7353778.

[3] L. Birglen and C. Gosselin. Grasp-state plane analysis of two-phalanx underactuated fingers. Mechanism and Machine Theory, 41(7):807-822, 2006.

[4] L. Birglen, T. Laliberté, and C. Gosselin. Underactuated Robotic Hands. Springer, New-York, 2008. ISBN 9783540774587.

[5] Jean-Michel Boucher and Lionel Birglen. Performance augmentation of underactuated fingers' grasps using multiple drive actuation. Journal of Mechanisms and Robotics, 9(4):041003, 2017.

[6] R. Carpenter, R. Hatton, and R. Balasubramanian. Comparison of Contact Capabilities for Underactuated Parallel Jaw Grippers for Use on Industrial Robots. In 2014 ASME International Design Engineering Technical Conferences, Buffalo, NY, USA, August 17202014.

[7] M.G. Catalano, G. Grioli, E. Farnioli, A. Serio, C. Piazza, and A. Bicchi. Adaptive synergies for the design and control of the Pisa/IIT SoftHand The International Journal of Robotics Research, 33(5):768-782, 2014. doi: 10.1177/0278364913518998. URL http://dx.doi.org/10. $1177 / 0278364913518998$

[8] A. M. Dollar and R. D. Howe. A robust compliant grasper via shape deposition manufacturing. IEEE/ASME Transactions on Mechatronics, 11(2):154-161, April 2006. ISSN 1083-4435. doi: 10.1109/TMECH.2006. 871090.

[9] G. Franchi, A. ten Pas, R. Platt, and S. Panzieri. The Baxter Easyhand: A robot hand that costs \$150 US in parts. In Intelligent Robots and Systems (IROS), 2015 IEEE/RSJ International Conference on, pages 29172922, Sept 2015. doi: 10.1109/IROS.2015.7353779.

[10] S. Hirose and Y. Umetani. The development of soft gripper for the versatile robot hand. Mechanism and Machine Theory, 13:351-358, 1978.

[11] ISO/TS 15066:2016, Robots and robotic devices - Collaborative robots, International Organization for Standardization. Standard, 2016.

[12] G. A. Kragten, M. Baril, C. Gosselin, and J. L. Herder. Stable precision grasps by underactuated grippers. IEEE Transactions on Robotics, 27(6):1056-1066, Dec 2011. ISSN 1552-3098. doi: 10.1109/TRO.2011.2163432.

[13] Gert A. Kragten and Just L. Herder. The ability of underactuated hands to grasp and hold objects Mechanism and Machine Theory, 45(3):408 - 425, 2010. ISSN 0094-
$114 \mathrm{X}$.

[14] Gert A Kragten, H Allert Bosch, Teunis van Dam, Johan A Slobbe, and Just L Herder. On the effect of contact friction and contact compliance on the grasp performance of underactuated hands. In ASME 2009 International Design Engineering Technical Conferences and Computers and Information in Engineering Conference, pages 871-878. American Society of Mechanical Engineers, 2009.

[15] J. M. McCarthy. Geometric Design of Linkages. Springer-Verlag, 2000. ISBN 0-387-98983-8.

[16] R. Ozawa, K. Hashirii, and H. Kobayashi. Design and control of underactuated tendon-driven mechanisms. In Robotics and Automation, 2009. ICRA '09. IEEE International Conference on, pages 1522-1527, May 2009. doi: 10.1109/ROBOT.2009.5152222.

[17] G. Palli, U. Scarcia, C. Melchiorri, and G. Vassura. Development of robotic hands: The UB hand evolution. In 2012 IEEE/RSJ International Conference on Intelligent Robots and Systems, pages 5456-5457, Oct 2012. doi: 10.1109/IROS.2012.6386303. 